\title{
Pediatric-adolescent andrology: Single centre experience
}

\author{
Nicola Zampieri, Francesco Camoglio \\ Woman \& Child Hospital, Department of Surgery, Dentistry, Paediatrics and Gynecology; Division of Pediatric Surgery, \\ University of Verona, Italy.
}

\begin{abstract}
Summary Introduction: Andrology is the medical specialty dealing with men's health and reproductive system from birth to adulthood, including genital, hormonal, reproductive, sexual as well as psychological aspects; the aim of this study is to report our 10 year-experience Material and methods: In September 2009, a Pediatric Andrology Outpatient Clinic was opened at the Authors' Institution. The continuous request for access to the service, together with an increasingly helpful collaboration with local clinicians, has led to an increase in the number of treated patients. At the Clinic, visits are performed for both surgical and medical consultations by the multidisciplinary medical group for the treatment of conditions in the adolescent patient. All patients are followed every 3, 6 and 12 months when indicated. Patients with undescended testes were excluded because managed into a specific protocol. Also patients with syndrome or metabolic diseases are excluded from the analysis.

Results: During the study period, September 2009-September 2019, the following conditions were managed: varicocele 1436 patients; gynecomastia - 18 patients; penile curvature 89 patients; webbed penis - 132 patients; hypospadias-related diseases - 39 patients; erectile dysfunction - 14 patients; obesity and abnormal semen analysis - 47 patients. During the study period there was an increase for each category especially for medical reasons.

Conclusions: Pediatric-adolescent andrology clinics should count on the expertise of different skilled professionals to cope with an ever-increasing number of requests and to offer the timely management of conditions that until very recently were considered social taboos or caused concern only in adulthood like the erectile dysfunction. The evolution of our society, which also means evolution of the mass media, should go hand in hand with the development of Medicine, which needs to adjust to and prevent new healthcare issues.
\end{abstract}

KEY WORDS: Pediatric andrology; Varicocele; Undescended testes; Adolescent.

Submitted 7 January 2020; Accepted 28 January 2020

\section{INTRODUCTION}

Andrology is the medical specialty dealing with men's health and reproductive system from birth to adulthood, including genital, hormonal, reproductive, sexual as well as psychological aspects (1).

Regular andrological check-ups are essential to reveal possible problems and to receive thorough advice and information so that sexual and reproductive functions are well preserved.

Estimates report that about one in three males suffers from andrological diseases with rates varying according to the age: $27-30 \%$ of pediatric male subjects have reproductive and/or sexual conditions, especially cryptorchidism, varicocele, hypogonadism, congenital anomalies of the genitourinary tract and sexually transmitted diseases; in adulthood, $40 \%$ of men are affected by andrological diseases, in particular infertility and sexual problems. The main surgically correctable diseases to prevent hypofertility are varicocele (30\%) and undescended testes $(<5 \%)(1-3)$.

The origin of many of the andrological conditions appearing during adulthood is to be traced before the age of 18 years and sometimes even during gestation.

The male gonad is extremely sensitive to external events even during gestation and soon after childbirth up until puberty (4-5).

The andrological evaluation of pediatric patients is therefore extremely important for an early diagnosis of genital anomalies such as penile alterations or abnormal positions of the testis; early evaluation is helpful also to search for risk factors in terms of male general and sexual health.

The aim of this study was to report the Authors' 10-year experience at the Pediatric Andrology Outpatients clinic of their Institution, focusing on the different aspects of surgically treated andrological diseases and on the type of andrological requests.

\section{Materials AND METHOdS}

In September 2009, a Pediatric Andrology Outpatients Clinic was opened at the Institution of Authors. The continuous request for access to the service, together with an increasingly helpful collaboration with local clinicians, has led to an increase in the number of treated patients.

At the Clinic, visits are performed both for surgical conditions and for medical consultations when required by the multidisciplinary medical group for the treatment of conditions in the adolescent patient.

Through the review of the cases, the aim was to focus on the type of conditions and the ever-increasing requests for advice and treatment. The study excluded the patients with phimosis, undescended testes and hypospadias, although it included penile complications such as residual penile curvatures or requests for penile lengthening. Upon specific request, some patients had hormonal tests and semen analysis performed. At the Authors' Clinic, any patient who has accomplished his pubertal stage can perform semen analysis, even though he is a minor, provided that an informed consent has been signed by the patient together with his referring physician and his par- 
ents. For undescended testes the Clinic adopts a specific follow-up procedure, which is not always performed by the same medical team (6).

Other diseases, such as Klinefelter, metabolic diseases or oncologic conditions are managed with a multidisciplinary approach. Depending on the different conditions, patients are followed every 3, 6 or 12 months.

The Clinic medical team includes 2 senior pediatric surgeons ( 1 with high specialty in andrology and urology), 2 registrars and 1 pediatric radiologist.

\section{RESULTS}

During the study period, September 2009-September 2019, the following conditions were managed: varicocele - 1436 patients; gynecomastia - 18 patients; penile curvature - 89 patients; webbed penis - 132 patients; hypospadias-related diseases - 39 patients; erectile dysfunction 14 patients; obesity and abnormal semen analysis - 47 patients. Patients data and distribution are showed into Table 1.

Varicocele: 548 patients during the study period received surgery with different techniques depending on the type of reflux (7-8).

Of these, 278 performed a spermiogram. Once the pubertal development was accomplished, non-surgical patients were always offered to perform a follow-up spermiogram. Gynecomastia: surgery was mainly requested for cosmetic reasons; only 18 cases out of the 69 managed at the Clinic showed concomitant hyperplasia of the gland. In 1 case, carcinoma was diagnosed in situ.

Penile curvature: 89 patients; 27 had a lateral curvature between 60 and 90 degrees, 18 between 45 and 60 degrees and the remaining 44 had a curvature of less than 45 degrees. These included 15 ventral curvatures, 5 dorsal curvatures and 24 lateral curvatures. In 10 patients, exeresis of the traction plate with the addition of biological material resolved the penile curvature.

Webbed penis: all patients received surgery after one year from birth; in 24 patients the suprapubic fat was removed in addition to the reconstruction of the penoscrotal and penopubic junctions.

Hypospadias-related diseases: 39 patients; 15 had a residual ventral curvature between 15 and 30 degrees; the remaining patients required lengthening at puberty, with exeresis of the suspensory ligament. In two cases (pubertal patients) the diameter was increased with the addition of biological material.

\section{Table 1.}

Patient distribution.

\begin{tabular}{|c|c|c|c|c|}
\hline $\begin{array}{l}\text { Disease } \\
\text { varicocele }\end{array}$ & $\begin{array}{c}\text { Number of pts } \\
1436\end{array}$ & $\begin{array}{l}\text { Age range } \\
13 \pm 2.1\end{array}$ & $\begin{array}{c}2009-2014 \\
727 \\
184 \text { underwent surgery }\end{array}$ & $\begin{array}{c}2015-2019 \\
1436 \\
364 \text { underwent surgery }\end{array}$ \\
\hline Gynecomastia & 18 & $14 \pm 0.9$ & 7 & 11 \\
\hline Penile curvature & 89 & $14 \pm 3.7$ & 24 & 65 \\
\hline Webbed penis & 132 & $5 \pm 1.8$ & 59 & 73 \\
\hline Hypospadias related disease & 39 & $13 \pm 3.1$ & 12 & 27 \\
\hline E.R. & 14 & $16 \pm 2.1$ & 1 & 13 \\
\hline Obesity & 47 & $17 \pm 1.2$ & 2 & 45 \\
\hline
\end{tabular}

Erectile dysfunction: none of these patients had a concomitant surgical condition. 12 patients reported the problem after the age of 15 years, after the first sexual intercourse was reported as being unsatisfactory. Two patients over the age of 17 years made a continuous use of drugs. This group of patients included two male subjects over the age of 16 years who suffered from oncological conditions and had received a transplant.

Obesity and abnormal semen analysis: all the patients accessed the Clinic mainly for the evaluation of the size of their external genitalia; all the patients were then offered to perform spermiogram.

\section{Follow-up}

During the study period there was an increasing number of requests, with an increasing percentage of those patients requiring medical or aesthetic check. Focusing on each specific field, there was a statistical significance increasing percentage between the year 2009-2014 and 2015-2019 ( $<$ < 0.05).

Excluding varicocele group where the percentage is stable during year (range between 475 and 576 visit per year with) the most significance differences were found into the penile curvature group (24 vs 65 cases), hypospadias related disease (12 vs 27 cases) and erectile dysfunction ( 1 vs 13 cases) $(\mathrm{p}<0.05)$.

About webbed penis there was an increasing demand but without statistical differences (59 vs 73 cases) probably because the main request was for phimosis and the final diagnosis for webbed penis. Obesity group increased after our National register study about bariatric surgery in adolescent and for this reason it was not possible to add any data about the increasing requests respect to other subgroups (9).

In all cases of varicocele, testicular hypotrophy remains the main indication for surgery. As reported in previous studies, all these patients had spontaneous continuous spermatic vein reflux (10-11).

They were corrected according to their pubertal stage and a correlation was found between high FSH level and small ipsilateral testicular volume with the controls. For this group motility, morphology and sperm count were significantly reduced than for the patients with the same grade of varicocele and spermatic vein reflux who were treated conservatively (normal testicular size). In terms of hormone levels, only FSH varied significantly in patients with different grades of testicular hypotrophy; it was not possible to observe the same for $\mathrm{LH}$, testosterone (TT), estradiol or prolactin levels, which were not different between operated and non-operated patients.

The patients who received post-op semen analysis after 1 year showed a statistically significant improvement in motility and morphology. Among non-operated patients, sperm morphology (head anomalies) was the main abnormal parameter, followed by motility and sperm count. These patients with abnormal semen analysis underwent surgery.

None of the patients with gynecomastia had abnormal hormone parameters, but for all the operated patients the cumula- 
tive testicular volume was smaller than for controls ( $\mathrm{p}<$ 0.05 ); also, the adolescents with gynecomastia had a testicular volume smaller than those with other andrological disease such as varicocele. Only 2 patients had semen analysis after surgery and the parameters were within the reference range.

In the penile curvature group, 89 patients, 27 had a lateral curvature between 60 and 90 degrees, 18 between 45 and 60 degrees and the remaining 44 had a curvature of less than 45 degrees. These included 15 ventral curvatures, 5 dorsal curvatures and 24 lateral curvatures. In 10 patients, exeresis of the traction plate with the addition of biological material resolved the penile curvature. The increased request trough the period was probably related to a preventative campaign at school where our team each year performed 2-hour meetings with adolescents focusing about andrological disease. The same is done for female with the gynecologists.

All the 132 patients with webbed penis had a surgical reconstruction of the penoscrotal junction and circumcision in case of tight phimosis; 24 patients had removal of suprapubic fat with a semi-circular incision in addition to the reconstruction of the penoscrotal and penopubic junctions. At the end of the follow-up period, 4 patients (aged between 12 and 15 years) underwent varicocelectomy. So far, no sexually active patient has reported any functional problems. Even if the main indication for the first visit by the general pediatricians was phimosis, in the first 5 year period, during the years it is was possible to establish an increased request for this specific disease and not only was phimosis, due to the well-known disease by the general pediatricians.

The group of patients with complications related to the correction of hypospadias within 18 months from birth was certainly the most difficult to manage. As it is known, the possible complications related to urethroplasty include fistulas and their strictures/stenoses as well as any possible complications or discomfort later during adolescence. 39 patients are currently followed at the Clinic for residual ventral curvature between 15 and 30 degrees (15 patients) and for penile lengthening (5 patients aged between 15 and 18 years). 2 patients in this group also requested an increased penile diameter and were treated with a filler. The remaining 10 patients suffer from erection pain and 3 sexually active patients report pain during ejaculation. These last fields are treated and recognized especially in the second 5-year period, due to the multidisciplinary team approach.

Another group of patients, new in terms of type of condition, is represented by adolescents with erection problems. 14 patients from this group are currently followed at the Clinic, all over 15 years of age and sexually active. None has been diagnosed with an organic disease, although most of the patients reported some discomfort during intercourse and a feeling of inadequacy with the partner. It is important to notice that no patient reported erectile dysfunction during masturbation. Two patients reported the use and abuse of drugs and alcohol. In general, about $90 \%$ of the patients reported difficulties in maintaining the erection but not in its induction. Two further patients accessed the Clinic after receiving a transplant following a hematological disease. As reported in a previous study, after the clinical and radiological evaluation, they answered a questionnaire on the quality of their sexual life. Both were without relapses and reported a reduced quality of erection, especially regarding maintenance (12). This group of patients is the example of the transitional age defect: the age group 13-18 years in the past was never considered, while female are constantly followed by gynecologists.

The andrological evaluation and obesity in the pediatric-adolescent age is of recent interest; although this relation is well established for the adults, little has been done for the pediatric age group. The patients who are currently cared for at the Authors' Clinic mainly requested first access for a genital evaluation, especially for concealed penis.

The hormonal and semen analysis tests (performed on 14 patients out of 47) showed that the testicular volume of overweight/obese adolescents (BMI $>25)$ was smaller than in patients with a normal weight $(p<0.05)$. These patients also had a delay in pubertal development and a reduced quality of their semen (mainly indicated by morphologic abnormalities and reduced motility).

The study patients did not show abnormal hormone levels (FSH, LH, TT, Estradiol) even if the FSH level seems to be higher respect to normal weight patients.

\section{Discussion}

Andrology greatly trusts primary prevention to reduce the incidence of andrological diseases and conditions. Clinical studies and primary prevention policies in andrology should be focused on the most vulnerable crucial phases of male gonad development that can be affected by a variety of external agents. The preservation of the genital and sexual health of young people also means protecting their fertility potential, a very important action within the broader scope of the interventions aimed at reducing the drop in the birth rate which is currently affecting modern society (13-15).

It is therefore well established that most of the andrological problems have a prenatal origin and can be diagnosed, studied and treated before adulthood. As reported in a recent study, the prevalence of male adolescent that are followed by andrologists is very low, if compared to female; this could be a "gender" discrimination (16). Although the development of the gonads is complex and not yet fully understood, there are some factors that could interfere with their correct anatomical development as well as with the successful pubertal development of the subject. A placental malfunction is associated with an abnormal andrological development: several studies described a relation between fetal growth restrictions and an increased risk of male reproductive problems, including hypospadias, cryptorchidism and testicular cancer (15-19).

In addition, twin or triplet pregnancy and preterm birth were shown to be associated with non-gestational impaired reproductive development. Again, an increased birth weight in males was also positively correlated with adult serum testosterone levels, while adult men born with lower birth weights were found to display features of hypogonadism, with reduced testicular size, lower 
testosterone levels and higher LH values than controls born with weights within the reference range. Other maternal factors can affect fertility: abnormal maternal glucose metabolism in pregnancy may be associated with an increased risk for genital malformation as well as maternal obesity, smoking, maternal serum estrogens, and estrogenic endocrine disruptor exposure (20-24).

This point in mind, in order to safeguard the reproductive and sexual health of young men, a synergic approach involving pediatricians, general practitioners, doctors at family planning clinics and andrologists for the adults plays a key role together with the implementation of territorial networks that may enhance the integration of the know-how and expertise of all these health professionals. This is especially true in the extremely vulnerable period of life generally ranging from 11 to 18 years of age, when young male patients experience the transition to adult life and maturity from a reproductive and sexual point of view.

If we exclude the cases treated for varicocele, which has a more consolidated follow-up, what emerged from the analysis of the cases treated at the Authors' Clinic was a progressive increase in some conditions which were not reported by patients until recent years. The problems related to urethroplasty, obesity, gynecomastia, webbed penis, and erectile dysfunction seem to be common causes of concern related more to the adolescents' increased awareness and sexual maturity than to real healthcare issues suggested by a territorial primary prevention system.

Two aspects deserve particular attention: the increase in obesity and in sexual dysfunctions in the adolescents can be considered important risk factors for the andrological health of adults. The merely functional aspect of the erectile dysfunction, if unrelated to conditions of surgical interest (outcome of penile surgery, bladder surgery, anorectal malformations, etc.), is complex to manage. Many adolescents experience pubertal development, relationship with their body and with their partners in an increasingly confused context, deeply affected by complex social stereotypes. A recent study pointed out that the main problems reported in addition to pain during intercourse were erectile dysfunction and premature ejaculation (25-27).

This latter aspect is difficult to be properly evaluated, since very often it is not clear what teenagers mean by "premature" ejaculation and if the real situation clashes with their expectations, or with what is considered as acceptable by their friends, by their readings on the internet or on the social media, as reported by many patients who accessed the Authors' Clinic. A different approach should be used in case of patients with erectile dysfunction associated with a history of cancer. In fact, despite being disease-free, these patients feel discomfort since they consider themselves as cancer patients, as being "different". In the Authors' experience, these patients are more easily treated because they have faster and more effective response mechanisms to the pathology and to their impaired physical condition than those young patients who have never experienced any pathological condition before (12).

Obesity in relation to male reproductive health is well recognized, especially in adulthood. Obesity alters fertility and the fertility potential through various mechanisms, mainly the reduced quantity of testosterone used and converted into estrogen by the adipocytes and the reduced quality of the seminal fluid. Although most of the obese adolescents are monitored for weight reduction primarily to reduce diabetes, hypertension, cardiac risk and orthopedic diseases, little action is taken in terms of fertility potential; in fact, for the obese patients the primary aim is weight loss to improve self-image, while their overall health status is kept in the background. The experience on adult patients treated both clinically and surgically for obesity showed a significant improvement in hormone levels and semen quality in those patients who obtained correct weight control, and this proved to be true for male and female patients alike (27-30).

The above considerations should drive patients towards weight control not only for esthetic and certainly important functional reasons, such as reducing hypertension, arthropathies and diabetes, but also to safeguard their fertility potential.

Prevention policies should be gender specific, with the awareness that if many actions are currently implemented for the prevention of female conditions, little is done for male patients. For young male adults, an objective examination to the genitalia, comprehensive hormone panel tests and a spermiogram can help the diagnosis and early treatment of many conditions that may affect male fertility potential.

Pediatric-adolescent andrology clinics should count on the expertise of different skilled professionals to cope with an ever-increasing number of requests and to offer the timely management of conditions that until very recently were considered social taboos or caused concern only in adulthood like the erectile dysfunction. The evolution of our society, which also means evolution of the mass media, should go hand in hand with the development of Medicine, which needs to adjust to and prevent new healthcare issues.

\section{REFERENCES}

1. Zampieri N, Bianchi F, Patanè S, Camoglio FS. Infertility worldwide: the lack of global pediatric andrologists and prevention. In Male Reproductive Health (Eds. Wei Wu, Francesco Ziglioli and Umberto Maestroni) doi: 10.5772/intechopen.88459.

2. Datta J, Palmer MJ, Tanton C, et al. Prevalence of infertility and help seeking among 15,000 women and men. Hum Reprod. 2016; 31: 2108-2118.

3. Agarwal A, Mulgund A, Hamada A, Chyatte MR. A unique view on male infertility around the globe. Reprod Biol Endocrinol. 2015; 13:37-46.

4. Juul A, Almstrup K, Andersson AM, et al. Possible fetal determinants of male infertility. Nat Rev Endocrinol 2014; 10:553-562.

5. Skakkebaek NE, Rajpert-De Meyts E, Main KM. Testicular dysgenesis syndrome: An increasingly common development aspects.Hum Reprod. 2001; 16:972-978.

6. Zampieri N, Caridha D, Patanè S, et al. Elastonosographic evaluation of the post-operative morpho-volumetric recovery of the gonad in the cryptorchid patient. Am J Clin Exp Urol. 2019; 7:182-187. 
7. Camoglio FS, Zampieri N. Varicocele treatment in paediatric age:relationship between type of vein reflux, surgical technique used and outcomes Andrologia. 2016:; 48:389-392.

8. Zampieri $N$, Zuin V, Corroppolo $M$, et al. relationship between varicocele grade, vein reflux and testicular growth arrest Pediatr Surg Int. 2008; 24:727-30.

9. Castellani R, Toppino M, Favretti F, et al. National survery fro bariatric procedures in adolescents:long time follow-up. J Pediatr Surg. 2017; 52:1602-1605.

10. Zampieri N, Corroppolo $M$, Zuin V, et al. longitudinal study of semen quality in adolescents with varicocele: to treat or not? Urology. 2007; 70:989-993.

11. Zampieri N, Zuin V, Corroppolo M, et al. Varicocele and adolescents: Semen quality after 2 different laparoscopic procedures. J Androl. 2007; 28:727-33.

12. Andreini A, Zampieri N, Costantini C, et al. Chronic graft versus host disease is associated with erectile dysfunction in allogenic hematopoietic stem cell transplant patients: a single center experience. Leuk Lymphoma. 2018; 21:1-4.

13. Brauner EV, Hansen AM, Doherty DA, et al. The association between in utero exposure to stressful life events during pregnancy and male reproductive function in a cohort of 20-year-old offspring: the Taine study. Hum Reprod. 2019; 34:1345-1355.

14. Bouty A, Ayers KL, Pask A, et al. The genetic and environmental factors underlying hypospadias. Sex Dev. 2015; 9:239-259.

15. Rae MT, Kyle CE, Miller DW, et al. The effects of undernutrition, in utero, on reproductive function in adult male and female sheep. Anim Reprod Sci. 2002; 72:63-71.

16. Olana S, Mazzilli R, Delfino M, et al. Adolescence and andrologist: An imperfect couple. Arch Ital Urol Androl. 2018; 90:208211.

17. Trabert B, Chodick G, Shalev V, et al. Gestational diabetes and the risk of cryptorchidism and hypospadias. Epidemiology. 2014; 25:152-153.

18. van der Zanden LF, van Rooij IA, Feitz WF, Fet al. Aetiology of hypospadias: A systematic review of genes and environment. Hum Reprod Update. 2012; 18:260-283.
19. Nordenvall AS, Frisen L, Nordenstrom A, et al. Population based nationwide study of hypospadias in Sweden, 1973 to 2009: Incidence and risk factors. J Urol. 2014; 191:783-789.

20. Guerrero-Bosagna CM, Skinner MK. Epigenetic transgenerational effects of endocrine disruptors on male reproduction. Semin Reprod Med. 2009; 27:403-408.

21. Hart RJ, Doherty DA, Mori TA, et al. Features of the metabolic syndrome in late adolescence are associated with impaired testicular function at 20 years of age. Hum Reprod. 2019; 34:389-402

22. Bonde JP, Flachs EM, Rimborg S, et al. The epidemiologic evidence linking prenatal and postnatal exposure to endocrine disrupting chemicals with male reproductive disorders: A systematic review and meta-analysis. Hum Reprod Update. 2016; 23:104-125.

23. Anway MD, Cupp AS, Uzumcu M, Skinner MK. Epigenetic transgenerational actions of ndocrine disruptors and male fertility. Science. 2005; 308:1466-1469

24. O'Sullivan LF, Byers ES, Brotto LA, et al. A Longitudinal study of problems in sexual functioning and related sexual distress among middle to late adolescents. J Adolesc Health. 2016; 59:318-324.

25. Wiggins A, Tsambarlis PN, Abdelsayed G, Levine LA. A treatment algorithm for healthy young men with erectile dysfunction. BJU Int. 2019; 123:173-179.

26. O'Sullivan LF, Brotto LA, Byers ES, et al. Prevalence and characteristics of sexual functioning among sexually experienced middle to late adolescents. J Sex Med. 2014; 11:630-41.

27. Wozniak SE, Gee LL, Wachtel MS, Frezza EE. Adipose tissue: The new endocrine organ? A review article. Dig Dis Sci. 2009; 54:1847-1856

28. Lima N, Cavaliere H, Knobel M, et al. Decreased androgen levels in massively obese men may be associated with impaired function of the gonadostat. Int J Obes Relat Metab Disord. 2000; 24:14331437.

29. Jensen TK, Andersson A-M, Jørgensen N, et al. Body mass index in relation to semen quality and reproductive hormones among 1,558 Danish men. Fertil Steril. 2004; 82:863-870.

30. Hammoud AO, Gibson M, Peterson CM, et al. Obesity and male reproductive potential. J Androl. 2006; 27:619-626.

\section{Correspondence}

Nicola Zampieri, MD, PhD (Corresponding Author)

nicola.zampieri@aovr.veneto.it

Francesco Saverio Camoglio, MD, Prof

francesco.camoglio@univr.it

Pediatric Surgical Unit, Azienda Ospedaliera Universitaria Integrata

Piazzale Aristide Stefani 1, 37100 - Verona (Italy) 